\title{
Correction to: Strategic crossing of biomass and harvest index-source and sink-achieves genetic gains in wheat
}

\author{
Matthew P. Reynolds • Alistair J. D. Pask • William J. E. Hoppitt • \\ Kai Sonder · Sivakumar Sukumaran • Gemma Molero • Carolina Saint Pierre • \\ Thomas Payne • Ravi P. Singh • Hans J. Braun • Fernanda G. Gonzalez • \\ Ignacio I. Terrile $\cdot$ Naresh C. D. Barma $\cdot$ Abdul Hakim $\cdot$ Zhonghu He $\cdot$ \\ Zheru Fan • Dario Novoselovic • Maher Maghraby • Khaled I. M. Gad • \\ ElHusseiny G. Galal • Adel Hagras • Mohamed M. Mohamed • Abdul Fatah A. Morad • \\ Uttam Kumar • Gyanendra P. Singh • Rudra Naik • Ishwar K. Kalappanavar • \\ Suma Biradar - Sakuru V. Sai Prasad - Ravish Chatrath • Indu Sharma • \\ Kishor Panchabhai · Virinder S. Sohu • Gurvinder S. Mavi • Vinod K. Mishra • \\ Arun Balasubramaniam • Mohammad R. Jalal-Kamali • Manoochehr Khodarahmi • \\ Manoochehr Dastfal · Seyed M. Tabib-Ghaffari · Jabbar Jafarby • \\ Ahmad R. Nikzad • Hossein Akbari Moghaddam • Hassan Ghojogh • \\ Asghar Mehraban • Ernesto Solís-Moya • Miguel A. Camacho-Casas • \\ Pedro Figueroa-López · Javier Ireta-Moreno · Jorge I. Alvarado-Padilla • \\ Alberto Borbón-Gracia • Araceli Torres • Yei Nayeli Quiche • Shesh R. Upadhyay • \\ Deepak Pandey • Muhammad Imtiaz • Monsif U. Rehman - Manzoor Hussain • \\ Makhdoom Hussain • Riaz Ud-Din • Maqsood Qamar • Muhammad Sohail • \\ Muhammad Y. Mujahid • Gulzar Ahmad • Abdul J. Khan • Mahboob A. Sial • \\ Pompiliu Mustatea $\cdot$ Eben von Well $\cdot$ Moses Ncala $\cdot$ Stephan de Groot • \\ Abdelraheem H. A. Hussein • Izzat S. A. Tahir • Amani A. M. Idris • \\ Hala M. M. Elamein • Yann Manes • Arun K. Joshi
}

Received: 19 January 2017 / Accepted: 13 October 2017/Published online: 14 December 2017

(C) The Author(s) 2017. This article is an open access publication

Electronic supplementary material The online version of this article (https://doi:10.1007/s10681-017-2040-z) contains supplementary material, which is available to authorized users.

This article is part of the Topical Collection on Plant Breeding: the Art of Bringing Science to Life. Highlights of the 20th

EUCARPIA General Congress, Zurich, Switzerland,

29 August-1 September 2016

Edited by Roland Kölliker, Richard G. F. Visser, Achim Walter \& Beat Boller

M. P. Reynolds $(\bowtie)$ · A. J. D. Pask · K. Sonder

S. Sukumaran · G. Molero · C. S. Pierre

T. Payne $\cdot$ R. P. Singh $\cdot$ H. J. Braun

International Maize and Wheat Improvement Center

(CIMMYT), Apdo, 6-641, 06600 Mexico, DF, Mexico

e-mail: M.Reynolds@cgiar.org
W. J. E. Hoppitt

Leeds University, Leeds, UK

F. G. Gonzalez · I. I. Terrile

Instituto Nacional de Tecnología Agropecuaria, Pergamino, Argentina 


\section{Correction to: Euphytica (2017) 213:257 https://doi.org/10.1007/s10681-017-2040-z}

The original article was corrected. Author Muhammad Kundi should instead read: Muhammad Sohail.
Open Access This article is distributed under the terms of the Creative Commons Attribution 4.0 International License (http:// creativecommons.org/licenses/by/4.0/), which permits unrestricted use, distribution, and reproduction in any medium, provided you give appropriate credit to the original author(s) and the source, provide a link to the Creative Commons license, and indicate if changes were made.

G. P. Singh

Indian Agricultural Research Institute, New Delhi, India

R. Naik · I. K. Kalappanavar · S. Biradar

University of Agricultural Sciences, Dharwad, India

S. V. Sai Prasad

Indian Agricultural Research Institute, Indore, India

Z. Fan

Xinjiang Academy of Agricultural Science, Wulumuqi,

China

D. Novoselovic

Agricultural Institute Osijek, Osijek, Croatia

M. Maghraby

CIMMYT, Sohag, Egypt

K. I. M. Gad · E. G. Galal · A. Hagras ·

M. M. Mohamed - A. F. A. Morad

Field Crops Research Institute, Cairo, Egypt

U. Kumar

CIMMYT BISA, Punjab, India
R. Chatrath · I. Sharma

Indian Institute of Wheat and Barley Research, Karnal, India

K. Panchabhai

Syngenta India Ltd., Karnal, India

V. S. Sohu · G. S. Mavi

Punjab Agricultural University, Ludhiana, India

V. K. Mishra - A. Balasubramaniam

Banaras Hindu University, Varanasi, India 
M. R. Jalal-Kamali CIMMYT, Tehran, Iran

M. Khodarahmi - M. Dastfal · S. M. Tabib-Ghaffari . J. Jafarby · A. R. Nikzad · H. A. Moghaddam Seed and Plant Improvement Institute, Karaj, Iran

H. Ghojogh · A. Mehraban

Dryland Agricultural Research Institute, Maragheh, Iran

E. Solís-Moya · M. A. Camacho-Casas .

P. Figueroa-López · J. Ireta-Moreno ·

J. I. Alvarado-Padilla · A. Borbón-Gracia Instituto Nacional de Investigaciones Forestales, Agrícolas y Pecuarias, Mexico, Mexico

\section{A. Torres · Y. N. Quiche \\ CIMMYT CENEB, Obregon, Mexico}

S. R. Upadhyay · D. Pandey

Nepal Agriculture Research Council, Bhairahawa, Nepal

M. Imtiaz · M. U. Rehman

CIMMYT, Islamabad, Pakistan
M. Hussain

Regional Agricultural Research Institute, Bahawalpur, Pakistan

M. Hussain

Wheat Research Institute, Ayub Agricultural Research Institute, Faisalabad, Pakistan

R. Ud-Din · M. Qamar · M. Sohail · M. Y. Mujahid Crop Sciences Research Institute, National Agricultural Research Council, Islamabad, Pakistan

G. Ahmad

Cereal Crop Research Institute, Nowshera-Pirsabak, Pakistan

A. J. Khan

Nuclear Institute for Food and Agriculture,

Tarnab-Peshawar, Pakistan

M. A. Sial

Nuclear Institute of Agriculture, Tando-Jam, Pakistan 
P. Mustatea

National Agricultural Research and Development

Institute, Fundulea, Romania

E. von Well · M. Ncala

Small Grain Institute, Bethlehem, South Africa

S. de Groot

Sensako Pty Ltd, Bethlehem, South Africa

A. H. A. Hussein - I. S. A. Tahir - A. A. M. Idris ·

H. M. M. Elamein

Agricultural Research Corporation, Wad Medani, Sudan

Y. Manes

Syngenta, Paris, France

A. K. Joshi

CIMMYT, Kathmandu, Nepal 